\title{
Microwave synthesis and sintering characteristics of $\mathrm{CaCu}_{3} \mathrm{Ti}_{4} \mathrm{O}_{12}$
}

\author{
P THOMAS $^{\dagger, *}$, L N SATHAPATHY ${ }^{\dagger \dagger}$, K DWARAKANATH ${ }^{\dagger}$ and K B R VARMA* \\ *Materials Research Centre, Indian Institute of Science, Bangalore 560 012, India \\ ${ }^{\dagger}$ Dielectric Materials Division, Central Power Research Institute, Bangalore 560 080, India \\ ${ }^{\dagger \dagger}$ Ceramic Technological Institute, Bharat Heavy Electrical Limited, Bangalore 560 012, India
}

MS received 19 July 2007

\begin{abstract}
CaCu}_{3} \mathrm{Ti}_{4} \mathrm{O}_{12}$ (CCTO) was synthesized and sintered by microwave processing at $2.45 \mathrm{GHz}, 1 \cdot 1 \mathrm{~kW}$. The optimum calcination temperature using microwave heating was determined to be $950^{\circ} \mathrm{C}$ for 20 min to obtain cubic CCTO powders. The microwave processed powders were sintered to $94 \%$ density at $1000^{\circ} \mathrm{C} / 60 \mathrm{~min}$. The microstructural studies carried out on these ceramics revealed the grain size to be in the range 1-7 $\mu \mathrm{m}$. The dielectric constants for the microwave sintered $\left(1000^{\circ} \mathrm{C} / 60 \mathrm{~min}\right)$ ceramics were found to vary from $11000-7700$ in the $100 \mathrm{~Hz}-100 \mathrm{kHz}$ frequency range. Interestingly the dielectric loss had lower values than those sintered by conventional sintering routes and decreases with increase in frequency.
\end{abstract}

Keywords. Microwave processing; sintering; giant dielectrics; microstructure.

\section{Introduction}

Electroceramics associated with giant dielectric constants have been in increasing demand owing to the miniaturization of electronic devices. Recently, $\mathrm{CaCu}_{3} \mathrm{Ti}_{4} \mathrm{O}_{12}$ (CCTO), a perovskite based centrosymmetric material, has received much attention because of its high dielectric constant over a wide range of frequencies and temperatures (Subramanian et al 2000) and has a potential for internal barrier layer capacitor applications. The processing conditions were found to affect the dielectric behaviour of CCTO to a great extent (Subramanian et al 2000; Homes et al 2001; Adams et al 2002; Sinclaire et al 2002; Lunkenheimer et al 2004; Bender and Pan 2005; Sriprakash and Varma 2006). CCTO was generally prepared via conventional solid-state reaction route using $\mathrm{CaCO}_{3}$, $\mathrm{TiO}_{2}$ and $\mathrm{CuO}$ as starting materials in stoichiometric ratio. The mixture of these was calcined at high temperature $\left(>1000^{\circ} \mathrm{C}\right)$ for prolonged periods $(24-48 \mathrm{~h})$ with repeated intermediate grindings (Subramanian et al 2000; Lunkenheimer et al 2002, 2004; Sinclair et al 2002; Sriprakash and Varma 2006).

It was known in the literature that chemical reactions could be accelerated by microwave irradiation of reactants (Sutton 1989; Katz 1992; Landry et al 1995). Microwave heating is a novel technique for processing ceramic materials with many advantages such as uniform heating, rapid heating rate, considerably reduced processing time and temperature, improved material properties and performance. Many researchers have reported the enhance-

*Author for correspondence (kbrvarma@mrc.iisc.ernet.in) ment in the solid state reaction rate or the solid state diffusion rate in a microwave dielectric field (Rao et al 1999; Thakur et al 2002; Vaidhyanathan et al 2002; Satapathy et al 2005). Complex oxides such as cuprates (Baghurst et al 1988), vanadates (Vaidhyanathan et al 1995), tungstates (Baghurst and Mingos 1988), ferrites (Krage 1981), niobates and titanates (Vaidhyanathan et al 1997) were prepared by microwave assisted solid state synthesis.

Electromagnetic waves interact with the ceramic materials, leading to volumetric heating by the dielectric loss mechanism. There are two main physical loss mechanisms: the flow of conductive current (in particular, ionic conduction), and dipolar reorientation. Mathematically, both these losses may be included in an effective dielectric loss factor,

$$
\varepsilon_{\mathrm{e}}^{\prime \prime}=\varepsilon^{\prime \prime}+\frac{\sigma}{\omega \varepsilon_{0}},
$$

where $\varepsilon^{\prime \prime}$ is the dielectric loss factor, $\sigma$ the conductivity, $\varepsilon_{0}$ the permittivity of free space, $\omega$ the frequency, $\sigma / \omega \varepsilon_{0}$ represents the conductivity related losses. The power dissipated in the ceramics is given by

$$
P=\omega \varepsilon_{0} \varepsilon_{\mathrm{e}}^{\prime \prime}|E|^{2}=\omega \varepsilon_{0}\left(\varepsilon^{\prime \prime}+\frac{\sigma}{\omega \varepsilon_{0}}\right)|E|^{2},
$$

where $E$ is the electric field within the dielectric (Zhipeng et al 1999).

We thought that it was worth extending this technique for making ceramic powders of commercially important CCTO as this has moderately high dielectric loss which is one of the desired characteristics for efficient coupling. 
To the best of our knowledge, there exists no report so far on the microwave assisted solid-state synthesis of CCTO powders in the literature. A simple, relatively fast microwave assisted procedure for the synthesis of CCTO powders, their sintering behaviour along with preliminary dielectric data are reported in this communication.

\section{Experimental}

Stoichiometric amounts of high pure $\mathrm{CaCO}_{3}, \mathrm{TiO}_{2}$ and $\mathrm{CuO}$ were weighed and ball-milled using zirconia balls in an acetone medium for $2 \mathrm{~h}$. The resultant mixture was oven dried and subjected to microwave processing. The microwave equipment that was used was a modified kitchen microwave oven $(2.45 \mathrm{GHz}, 1.1 \mathrm{~kW})$ capable of generating temperatures up to $1600^{\circ} \mathrm{C}$ (Indian patent no. $1016 / \mathrm{del} / 2001)$. The starting mixture of reactants $(10 \mathrm{~g})$ was heated at $900^{\circ} \mathrm{C}$ and $950^{\circ} \mathrm{C}$ for various durations using the microwave oven. For this, the mixture was taken in a recrystallized alumina crucible and in turn kept inside the alumina-silicon carbide crucible covered with a microwave compatible casket which was made up of alumino-silicate blanket. Sheathed $\mathrm{Pt} / \mathrm{Rh}$ thermocouple was placed on the sample to monitor the temperature. The schematic of the casket used for calcinations is shown in figure 1. The microwave oven was switched off immediately after the specified soaking time and the calcined powder was removed from the oven after $2 \mathrm{~h}$ of experiment. The powders prepared via the microwave heating was cold pressed into pellets of $12 \mathrm{~mm}$ in diameter and $3 \mathrm{~mm}$ in thickness using $3 \%$ poly vinyl alcohol (PVA) and $1 \%$ polyethylene glycol as binders. The green pellets were then sintered using microwave oven at $1000^{\circ} \mathrm{C}$ for two different durations (30 and $60 \mathrm{~min}$ ). For comparison, the CCTO was also

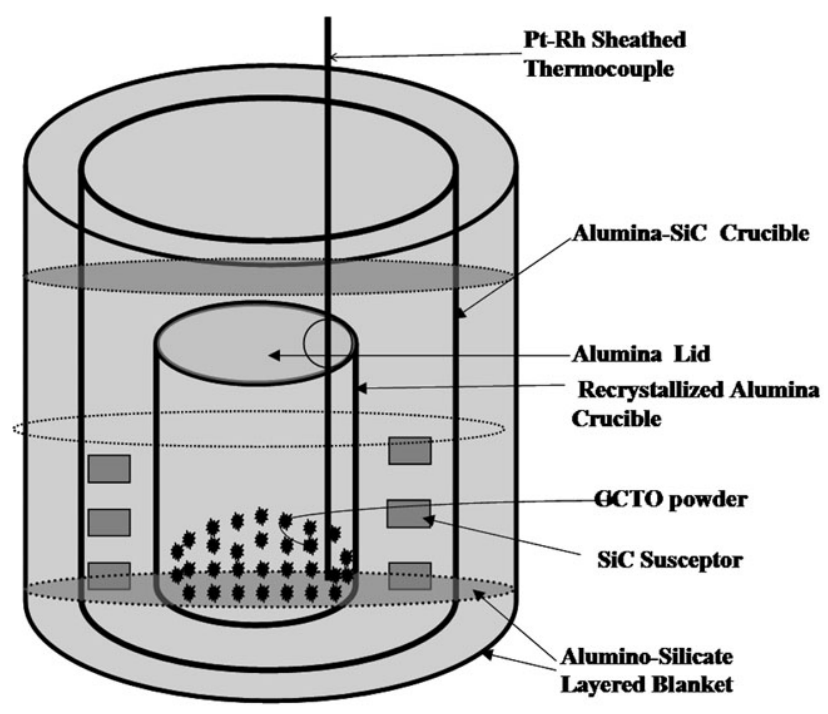

Figure 1. Schematic of set up used for calcining and sintering CCTO ceramic in this study. synthesized via the conventional solid-state reaction route by calcining the starting mixture at 950 and $1000^{\circ} \mathrm{C}$, respectively.

The formation of the monophasic compounds was confirmed via X-ray powder diffraction (XRD) studies. The densities of the sintered pellets were measured by the Archimedes principle using xylene as the liquid medium. Scanning electron microscope (SEM) (Cambridge Stereoscan S-360) was employed to study the microstructure of the sintered pellets. The capacitance measurements of the electroded pellets were carried out as a function of frequency (100 Hz-1 MHz) using an impedance gain-phase analyser (HP4194A).

\section{Results and discussion}

The XRD powder patterns for the microwave synthesized (at 900 and $950^{\circ} \mathrm{C}$ for different durations) are depicted in figure $2(\mathrm{a}-\mathrm{e})$. The $\mathrm{XRD}$ patterns corresponding to the powders that are microwave processed at $900^{\circ} \mathrm{C}$ (figure $2(\mathrm{a}-\mathrm{c}))$ show the presence of unreacted phases irrespective of the processing duration, whereas the XRD patterns corresponding to the powders processed at $950^{\circ} \mathrm{C}$ for 20 min (figure 2(e)) established the formation of single phasic CCTO. The X-ray diffraction patterns obtained for the powder processed by conventional heating at $950^{\circ} \mathrm{C}$ for different durations are shown in figure $3(\mathrm{a}-\mathrm{d})$. It is evident that the peaks corresponding to unreacted phases are present even after $10 \mathrm{~h}$ of calcination (figure $3(\mathrm{c})$ ) and phase-pure CCTO could be obtained only at $1000^{\circ} \mathrm{C} / 5 \mathrm{~h}$ (figure 3(d)). Thus, the microwave method of synthesizing CCTO is more favourable for obtaining phase pure powders of CCTO as this is found to be fast and energy efficient.

Figure 4(a and b) shows the scanning electron micrograph of the fractured surfaces obtained for the pellets

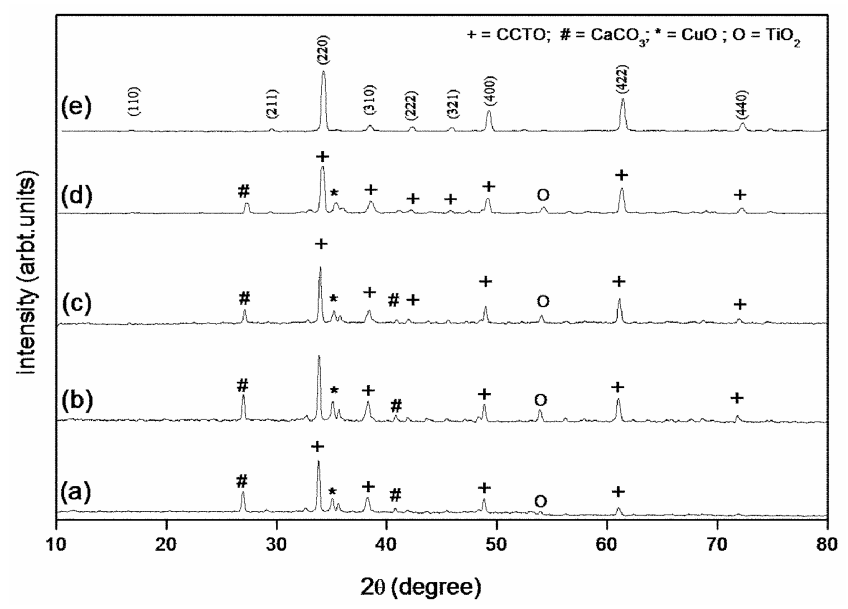

Figure 2. X-ray diffraction patterns for the powders synthesized in microwave furnace at (a) $900^{\circ} \mathrm{C} / 20 \mathrm{~min}$, (b) $900^{\circ} \mathrm{C} /$ $30 \mathrm{~min}$, (c) $900^{\circ} \mathrm{C} / 60 \mathrm{~min}$, (d) $950^{\circ} \mathrm{C} / 10 \mathrm{~min}$ and (e) $950^{\circ} \mathrm{C} /$ $20 \mathrm{~min}$. 
sintered at 30 and $60 \mathrm{~min}$. It is observed that there is no significant difference in the grain sizes between the pellets sintered at 30 and $60 \mathrm{~min}$. It establishes the presence of the grains with sizes lying in $2-7 \mu \mathrm{m}$ range in both the cases. As seen from the microstructure, the ceramic sintered at $60 \mathrm{~min}$ is denser than the $30 \mathrm{~min}$ sintered one and the density increased from $92-94 \%$.

Figure 5(a) shows the frequency $(100 \mathrm{~Hz}-100 \mathrm{kHz})$ dependence of room-temperature dielectric constant for the microwave sintered pellets at $1000^{\circ} \mathrm{C}$ for 30 and $60 \mathrm{~min}$. As seen from figure 5(a), the dielectric constant shows an increasing trend as the sintering duration increased from $30 \mathrm{~min}$ to $60 \mathrm{~min}$, whereas the dielectric loss shows a decreasing trend at any frequency under study. The dielectric constants for the microwave processed CCTO decrease with increase in frequency. Typically, it decreases from $11,000(100 \mathrm{~Hz})$ to $7700(100 \mathrm{kHz})$ in the case of $1000^{\circ} \mathrm{C} / 60 \mathrm{~min}$ sintered pellets while dielectric loss decreased from $0.58-0.081$ as the frequency increased from $100 \mathrm{~Hz}-100 \mathrm{kHz}$ (figure 5(b)). Adams et al (2002) proposed a direct link between grain size and permittivity based on two sets of samples obtained by varying the sintering time. In contrast to these results, the dielectric constants obtained in this work are not directly correlated to the grain size, which is in agreement with the results obtained by Seymen et al (2005). The observed higher value of dielectric constant for the pellet sintered at $1000^{\circ} \mathrm{C} / 60 \mathrm{~min}$ is ascribed to the enhanced density. The dielectric constants for CCTO ceramics obtained by the microwave sintering $\left(1000^{\circ} \mathrm{C} / 60 \mathrm{~min}\right)$ is higher than that of CCTO ceramics that were fabricated from the powders obtained by (i) solid state reaction route (Jha et al 2003; Seymen et al 2005; Ni et al 2006; Sriprakash and Varma 2006), (ii) polymer precursor route (Jha et al 2003) and (iii) modified glycine nitrate process (GNP) (Ren et al 2004). It is worth noting that the dielectric loss value in

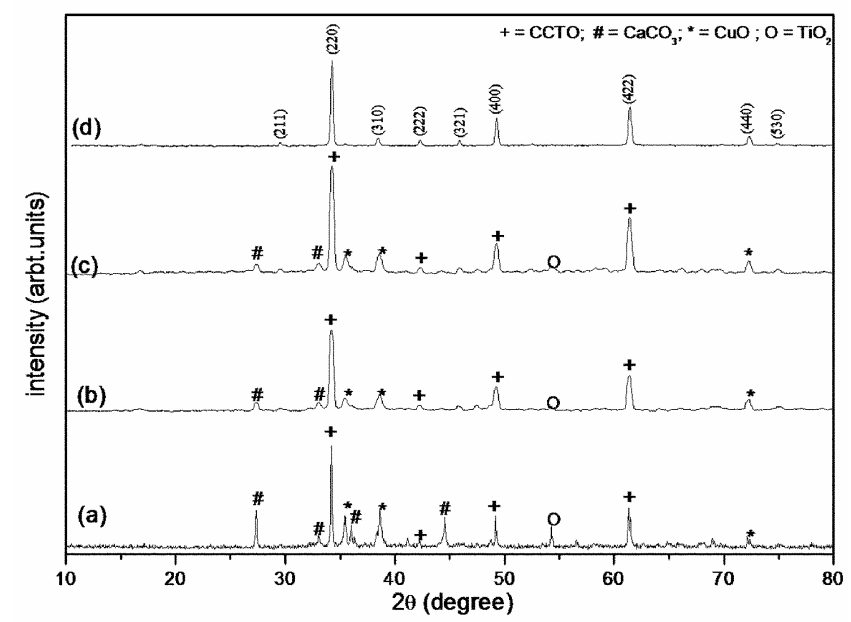

Figure 3. X-ray diffraction patterns for the conventional synthesized powder at: (a) $950^{\circ} \mathrm{C} / 60 \mathrm{~min}$, (b) $950^{\circ} \mathrm{C} / 6 \mathrm{~h}$, (c) $950^{\circ} \mathrm{C} / 10 \mathrm{~h}$ and $(\mathrm{d}) 1000^{\circ} \mathrm{C} / 5 \mathrm{~h}$. the whole frequency range of $1 \mathrm{kHz}-100 \mathrm{kHz}$ is below $0 \cdot 17$, which is lower than that of the results reported by Jha et al (2003) and Ren et al (2004). Further, the dielectric properties reported in this work are for the pellets that
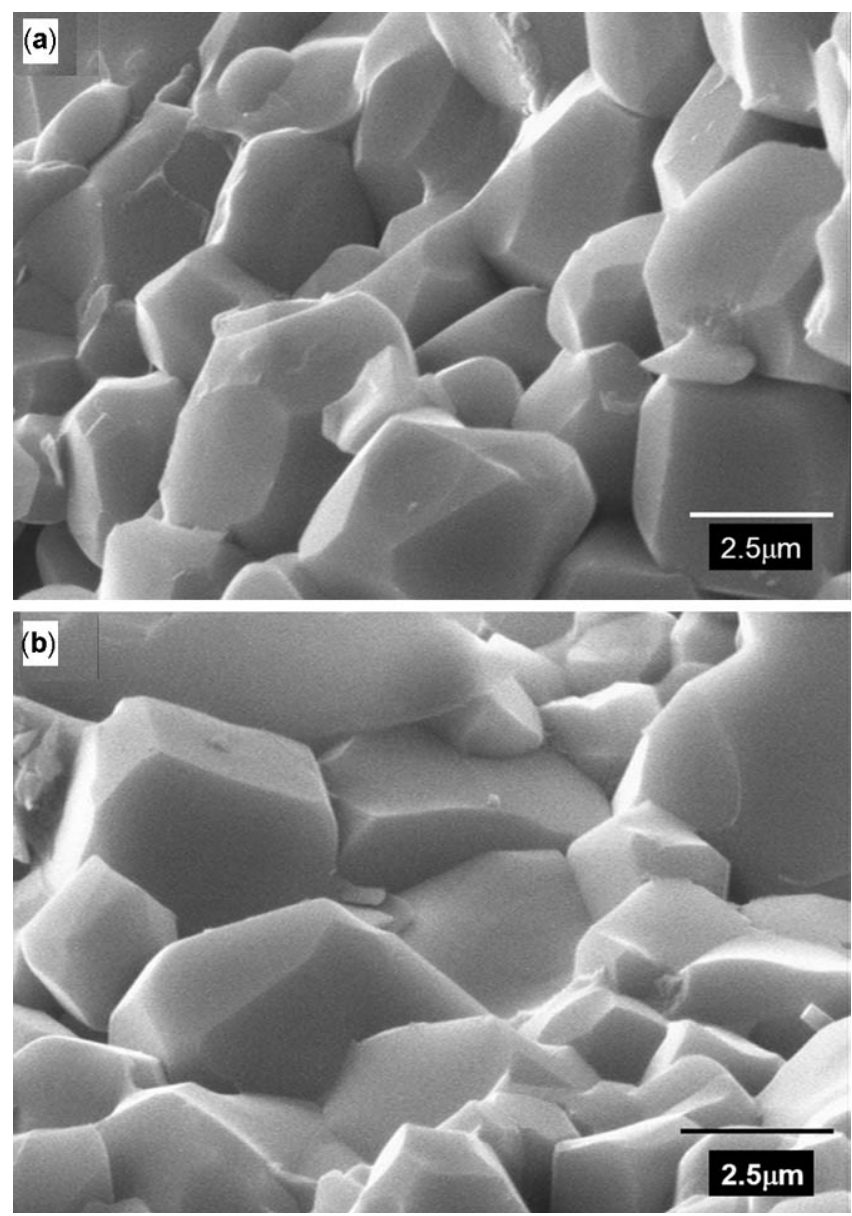

Figure 4. Scanning electron micrographs for microwave sintered pellets: (a) $1000^{\circ} \mathrm{C} / 30 \mathrm{~min}$ and (b) $1000^{\circ} \mathrm{C} / 60 \mathrm{~min}$.

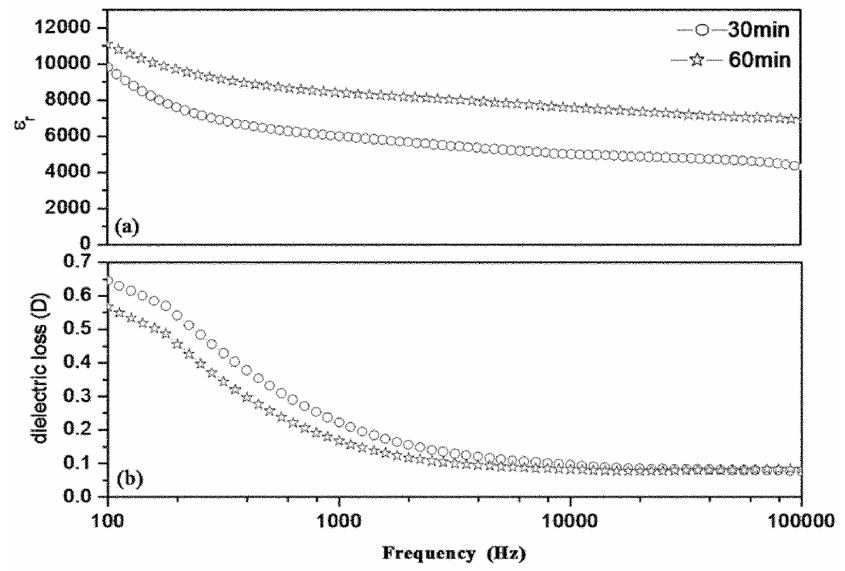

Figure 5. Frequency dependence of room-temperature dielectric properties for the microwave sintered pellet at $1000^{\circ} \mathrm{C}$ for 30 and $60 \mathrm{~min}$. 
were sintered in microwave oven for a much shorter duration (60 min) than those reported earlier (Jha et al 2003; Ren et al 2004; Seymen et al 2005; Ni et al 2006; Sriprakash and Varma 2006).

The incidence of giant dielectric constants in CCTO have been attributed to (i) the barrier layer capacitance arising at twin boundaries (Subramanian et al 2000), (ii) the interfaces between the sample and the electrode (Lunkenheimer et al 2002), (iii) grain boundary effects (Sinclair et al 2002; Liezhang 2005; Liu et al 2005), (iv) the lattice distortions (Chung 2005), (v) the internal domains (Fang and Liu 2005) and (vi) the oxygen vacancies ( $\mathrm{Li}$ et al 2004; Pires et al 2006). Though different explanations for the origin of high dielectric constant have been proposed, the real mechanism is still debatable as to whether it is intrinsic or extrinsic in nature. Since the electrical properties of ceramics are mainly dependent on the bulk as well as on the microstructure vis-à-vis processing conditions, which include sintering temperatures and atmospheres (Subramanian et al 2000; Homes et al 2001; Adams et al 2002; Sinclair et al 2002; Lunkenheimer et al 2004; Ren et al 2004; Bender and Pan 2005; Seymen et al 2005; Ni et al 2006; Sriprakash and Varma 2006), systematic work has been in progress to visualize the dielectric properties of the CCTO pellets sintered under various microwave sintering conditions.

\section{Conclusions}

The microwave method of synthesizing СCTO powders has been demonstrated. This is found to be fast and energy efficient as compared to that of the conventional methods. Indeed CCTO powders could easily be synthesized by using $2.45 \mathrm{GHz}, 1.1 \mathrm{~kW}$ modified kitchen microwave oven at $950^{\circ} \mathrm{C}$ for $20 \mathrm{~min}$. The dielectric maximum of 11,000 at $100 \mathrm{~Hz}$ accompanied by lower loss was obtained for the pellets sintered at $1000^{\circ} \mathrm{C}$ for $60 \mathrm{~min}$.

\section{Acknowledgement}

One of the authors (PT) would like to thank the Central Power Research Institute, Bangalore, for the financial support (CPRI Project No.5.4.49).

\section{References}

Adams T B, Sinclair D C and West A R 2002 Adv. Mater. 1418 Baghurst D R and Mingos M P 1988 J. Chem. Soc. Chem. Commun. 829
Baghurst D R, Chippindale A M and Mingos D M P 1988 Nature 332311

Bender B A and Pan M-J 2005 Mater. Sci. Engg. B117 339

Chung S Y 2005 Appl. Phys. Lett. 87052901

Fang T T and Liu C P 2005 Chem. Mater. 175167

Homes C C, Vogt T, Shapiro S M, Wakimoto S and Ramirez A P 2001 Science 293673

Indian patent on 'A continuous process control device for a kitchen microwave oven for use as a sintering furnace for sintering small size samples' (application number 1016/DEL/ 2001)

Jha P, Arora P and Ganguli A K 2003 Mater. Lett. 572443

Katz J D 1992 Ann. Rev. Mater. Sci. 22153

Krage M K 1981 Am. Ceram. Soc. Bull. 601232

Landry C C, Lockwood J and Barron A R 1995 Chem. Mater. 7 699

Leizhang 2005 Appl. Phys. Lett. 87022907

Li J, Cho K, Wu N and Ignatiev A 2004 IEEE Trans. Dielectr. Electr. Insul. 11534

Liu J, Duan C, Mei W N, Smith R W and Hardy J R 2005 J. Appl. Phys. 98093703

Lunkenheimer P, Bobnar V, Pronin A V, Ritus A I, Volkov A A and Loidl A 2002 Phys. Rev. B66 052105

Lunkenheimer P, Fichtl R, Ebbinbhaus S G and Loidl A 2004 Phys. Rev. B70 172102

Ni L, Chen X M, Liu X Q and Hou R Z 2006 Solid State Commun. 13945

Pires M A et al 2006 Phys. Rev. B73 224404

Rao K J, Vaidhyanathan B, Ganguli M and Ramakrishnan P A 1999 Chem. Mater. 11882

Ren W, Yu Z, Krstic V D and Mukherjee B K 2004 IEEE int. ultrasonic, ferroelectrics and frequency control, joint 50th annual conference, p. 149

Satapathy L N, Ramesh P D, Agrawal D K and Roy R 2005 Mater. Res. Bull. 401871

Seymen A, Tan X, Maria J -P and Cann D 2005 J. Elec. Ceram. 15203

Sinclair D C, Adams T B, Morrison F D and West A R 2002 Appl. Phys. Lett. 802153

Sriprakash B and Varma K B R 2006 Physica B382 312

Subramanian M A, Duran D, Li N, Reisner B A and Sleight A W 2000 Solid State Chem. 151323

Sutton W H 1989 Ceram. Bull. 68376

Thakur O P, Chandra Prakash and Agrawal D K 2002 Mater. Lett. 56970

Vaidhyanathan B, Ganguli M and Rao K J 1995 Mater. Res. Bull. 301173

Vaidhyanathan B, Raizada P and Rao K J 1997 J. Mater. Sci. Lett. 162022

Vaidhyanathan B, Agrawal D K, Shrout T R and Fang Yi 2002 Mater. Lett. 42207

Zhipeng X, Yang J, Huang X and Huang J 1999 J. Eur. Ceram. Soc. 19381 